\title{
La réglementation du travail à distance
}

\section{Elena Serebryakova}

\section{(2) OpenEdition}

Journals

Édition électronique

URL : https://journals.openedition.org/rdctss/750

DOI : $10.4000 /$ rdctss.750

ISSN : 2262-9815

Éditeur

Centre de droit comparé du travail et de la sécurité sociale

Édition imprimée

Date de publication : 1 avril 2021

Pagination : 182-185

ISSN : 2117-4350

\section{Référence électronique}

Elena Serebryakova, « La réglementation du travail à distance », Revue de droit comparé du travail et de la sécurité sociale [En ligne], 1 | 2021, mis en ligne le 01 avril 2021, consulté le 01 avril 2022. URL : http://journals.openedition.org/rdctss/750 ; DOI : https://doi.org/10.4000/rdctss.750

\section{(c) (i) (9)}

Revue de droit comparé du travail et de la sécurité sociale est mise à disposition selon les termes de la Licence Creative Commons Attribution - Pas d'Utilisation Commerciale - Pas de Modification 4.0 International. 


\title{
ELENA SEREBRYAKOVA
}

\author{
UNIVERSITÉ NATIONALE DE RECHERCHE, ÉCOLE DES HAUTES ÉTUDES \\ EN SCIENCES ÉCONOMIQUES »
}

\section{LA RÉGLEMENTATION DU TRAVAIL À DISTANCE}

L'année 2020 fut une année d'épreuves sanitaires, économiques et sociales pour la quasi-totalité des pays dans le monde. Nombre d'entre eux ont été contraints de prendre des mesures sévères afin de limiter la propagation de la pandémie de Covid-19. Ces mesures comprennent notamment le confinement, dont l'introduction a pu affecter la relation entre salariés et employeurs en raison de la distanciation des liens sociaux. Les organisations russes, même celles qui ne pratiquaient pas le travail à distance avant 2020, se sont empressées de mettre leurs salariés en télétravail. Pour autant, il n'était pas aisé de le faire dans les conditions de confinement, car le Code du travail de la Fédération de Russie ne prévoyait pas la modification du contrat pour un passage en télétravail. Cette lacune, comme d'autres, ont été corrigées par la loi du 8 décembre 2020ㄹ, entrée en vigueur le

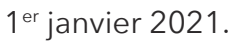

\section{I - TRAVAIL À DOMICILE, TÉLÉTRAVAIL ET TRAVAIL À DISTANCE}

Jusqu'au 1 er janvier 2021, le Code du travail prévoyait les termes de «travail à domicile» et de «télétravail»(distantsionnaya rabota). Le travail à domicile, contrairement au télétravail, ne nécessite pas l'utilisation de réseaux publics d'information et de communication pour son exécution et peut être effectué avec la participation éventuelle des membres de la famille du salarié. La notion de "travail à distance " (udaleaunnaya rabota) était absente du Code du travail, bien que dans la doctrine, ce terme ait été utilisé comme synonyme de télétravail².

Le ministère du Travail et de la Protection Sociale a provoqué une certaine confusion terminologique en avril 2020 lorsqu'il a recommandé aux employeurs de recourir à des formes flexibles d'emploi dans le cadre de la prévention de la propagation de la maladie de Covid $19^{3}$. Dans ces recommandations du ministère, le «travail à distance » est considéré comme une forme d'emploi distincte du travail à domicile et du télétravail. En même temps, le ministère n'a pas identifié les caractéristiques du travail à distance. II ressort toutefois de ces recommandations que, pour placer des salariés en situation de travail à distance, il suffit que l'employeur émette un ordre correspondant et le transmette aux salariés. Cette procédure était bien différente de celle du passage au travail à distance, au sens de télétravail, qui nécessitait la conclusion d'un avenant au contrat de travail avec chacun des salariés concernés par cette modification de l'organisation du travail.

1 Loi fédérale du 8/12/2020 n407-FZ sur les modifications du Code du travail de la Fédération de Russie concernant la réglementation du travail à distance et le transfert temporaire d'un employé à un travail à distance, à l'initiative de l'employeur et dans des cas exceptionnels.

2 Voir par exemple E. V. Kiseleva, «Le développement du travail à distance en Russie: avantages et inconvénients ", Izvestia AltSU, 2018, n6 (104) : https://doi.org/10.14258/izvasu(2018)6-30

3 Lettre du ministère du Travail et de la Protection sociale de la population de la Fédération de Russie, datée du 23 avril 2020. 
Depuis le 1er janvier 2021, l'expression «travail à distance » est inscrite dans le Code du travail. Elle est synonyme de télétravail. Le législateur distingue deux types de travail à distance: permanent et temporaire. Le télétravail permanent est effectué à domicile par un salarié, pendant toute la durée de validité du contrat de travail. Le travail temporaire à distance peut être effectué de manière continue à domicile durant une période ne dépassant pas 6 mois ou avec alternance de périodes de travail à domicile et sur site de l'entreprise.

\section{II - LA PROCÉDURE DE PASSAGE AU TÉLÉTRAVAIL}

Depuis le $1^{\text {er }}$ janvier 2021, la possibilité d'exercer les activités professionnelles à distance peut être prévue non seulement par un contrat de travail, mais aussi par un avenant à celui-ci, ou par une décision de l'employeur sans modification du contrat de travail. La conclusion, la modification et la résiliation du contrat de travail ou de l'avenant y relatif, sont possibles par la signature de documents sur papier ou par voie électronique. Dans ce dernier cas, l'employeur est strictement tenu d'utiliser une signature électronique authentifiée, tandis que le salarié peut utiliser une signature électronique plus simple à obtenir.

Un salarié peut également être placé temporairement en télétravail sur la base d'une décision prise par l'employeur en tenant compte de l'avis de l'instance élue de l'organisation syndicale de premier niveau. Il n'est pas nécessaire d'obtenir le consentement du salarié, il suffit de la lui faire connaître.

La décision de passage des salariés au télétravail peut être prise :

- dans des situations exceptionnelles, lorsqu'il existe une menace pour la vie ou susceptible d'affecter les conditions de vie de tout ou partie de la population (catastrophe naturelle, accident industriel, tremblement de terre, épidémies, épizootie...) ;

- En cas d'injonction d'une autorité gouvernementale ou municipale.

En cas de passage extraordinaire au travail à distance d'un salarié, aucune modification n'est apportée à son contrat de travail. Si, dans les cas susmentionnés, le passage du salarié en télétravail est impossible parce que son activité n'est pas éligible au télétravail, ou parce qu'il est impossible de lui fournir l'équipement, les logiciels et les outils de protection des données nécessaires, le salarié perçoit les indemnités de chômage technique dont le montant est égal aux $2 / 3$ du salaire de base du travailleur, sans compter les indemnités et les primes. II n'existe pas de plancher si bien que ces indemnités peuvent être inférieures au SMIC.

\section{III - L'EXÉCUTION DE L'ACTIVITÉ PROFESSIONNELLE PAR UN TRAVAILLEUR À DISTANCE}

Les relations entre le salarié et son employeur peuvent se faire par échange de documents qui ne nécessitent plus la signature électronique authentifiée obligatoire des parties liées par le contrat de travail. Elles peuvent choisir une autre forme de communication, en la stipulant dans le contrat de travail ou dans un avenant. Cette forme peut également être déterminée par un accord collectif ou par une décision de l'employeur prenant en compte l'avis d'une instance élue d'une organisation syndicale de premier niveau ${ }^{4}$.

4 Ces actes sont ci-après dénommés « actes normatifs locaux ». 
Le temps consacré aux communications électroniques entre le salarié et l'employeur est désormais inclus dans le temps de travail et, à ce titre, soumis à rémunération.

En règle générale, tous les équipements et installations nécessaires au travail d'un salarié à distance doivent être fournis par l'employeur. Pour effectuer ses activités professionnelles, le salarié a le droit d'utiliser les équipements, le matériel et les logiciels qui lui appartiennent, à condition que l'employeur ne s'y oppose pas. Les frais du salarié générés par l'utilisation de son équipement personnel sont remboursés par l'employeur selon la procédure et, dans la mesure prévue par l'accord entre les parties au contrat de travail, à la convention collective ou à un acte normatif local.

Un travailleur à distance n'a pas le droit de se rendre au bureau. Un salarié pour lequel le travail à distance est temporaire peut être sollicité par l'employeur pour exercer son activité sur son lieu de travail, dans les cas déterminés par le contrat de travail ou l'avenant, l'accord collectif ou l'acte normatif local. L'article 312.4 du Code du travail ne prévoit qu'une seule restriction : le travail à distance en situation d'urgence ne peut être interrompu par l'employeur.

\section{IV - LES MOTIFS SPÉCIFIQUES DE RÉSILIATION DU CONTRAT DE TÉLÉTRAVAIL}

La loi du 8 décembre 2020 a complété la liste des motifs de rupture du contrat de travail pour d'autres supplémentaires concernant uniquement les travailleurs à distance.

Un télétravailleur peut être licencié s'il ne communique pas avec l'employeur pendant plus de deux jours ouvrables consécutifs sans justification valable. Une période plus longue d'absence de contact avec l'entreprise peut être établie par les parties signataires au contrat de travail, par un accord collectif ou par un acte normatif local. Pour pouvoir licencier le salarié dans un tel cas, l'employeur doit respecter la procédure qui inclut la demande au salarié d'expliquer les raisons de la violation des règles et des conditions de relations établies avec l'employeur.

Le contrat de télétravail est susceptible d'être résilié si le salarié déménage, sous réserve de deux conditions simultanées:

1) un changement de lieu de résidence du salarié rend impossible l'exercice de ses tâches professionnelles dans les conditions précédentes. Par exemple, lorsqu'un décalage horaire à la suite d'une relocalisation empêche le travailleur de communiquer avec l'employeur à l'horaire précédemment fixé.

2) le salarié effectue en permanence un travail à distance. En effet, si le travail à distance est temporaire, le contrat de travail ne peut pas être résilié pour le motif en question. Dans ce cas, d'autres motifs de résiliation du contrat de travail s'appliquent.

\section{Conclusion}

Les modifications apportées au Code du travail par la loi du 8 décembre 2020 éliminent I'interprétation ambiguë des termes utilisés, simplifient le placement extraordinaire d'un salarié en télétravail, et apportent des garanties supplémentaires aux travailleurs à distance. Par conséquent, ces changements peuvent être évalués positivement. Pour autant, certains aspects du télétravail restent non résolus. 
Par ailleurs, les certaines dispositions de la loi du 8 décembre 2020 peuvent donner lieu à de nouvelles interprétations et donc à de nouveaux conflits potentiels du travail. En premier lieu, la loi prévoit qu'une partie importante des conditions du travail à distance peut être spécifiée dans un accord collectif ou un acte normatif local. Parmi ces éléments figurent la procédure et la forme de la relation et des conditions d'exercice du travail entre les parties signataires du contrat de travail, la procédure d'obtention des informations de l'employeur, le régime des heures de travail, la fréquence et la durée du travail à distance, les modalités et les montants de la compensation pour l'utilisation par le salarié de son équipement personnel pour raison professionnelle, etc.

Cette approche peut être considérée comme un élargissement du dialogue social. Toutefois, compte tenu du caractère facultatif de la conclusion d'accords collectifs et de leur impopularité, ainsi que l'absence d'organisations syndicales dans la plupart des entreprises russes, on peut considérer que les nouvelles règles aboutiront à une situation dans laquelle toutes les conditions du télétravail seront déterminées par l'employeur, de manière unilatérale. En effet, l'avis d'une instance élue d'une organisation syndicale de premier niveau ne peut être pris en compte que si une telle organisation existe dans une entreprise. En outre, la prise en compte de l'avis de l'instance élue n'est pas contraignante et n'implique pas que celle-ci ait le droit d'opposer son veto aux actes adoptés par l'employeur. Même si le syndicat est en désaccord avec une décision de l'employeur, cette dernière prend effet. La prise en compte de l'avis du syndicat est donc une procédure purement formelle.

De plus, en cas d'urgence, l'employeur peut placer un salarié en télétravail, ou rémunérer ses heures de chômage technique. Mais la loi ne fixe pas de critères qui permettent objectivement de déterminer s'il est possible ou non de fournir l'équipement nécessaire au salarié en mesure de télétravailler. En l'absence de tels critères, l'employeur peut abuser de ses prérogatives et mettre le salarié au chômage technique, même si son activité est " télétravaillable » ou si les conditions ne s'y opposent pas. La rémunération du temps passé au chômage technique aurait un coût significativement moindre pour l'employeur que celle du télétravail. Par conséquent, certains employeurs peuvent déclarer des temps de non travail effectif pour certains salariés, et demander à d'autres d'effectuer des tâches plus nombreuses et intenses à distance. Dans une telle situation, seul l'employeur est gagnant. On peut supposer que, dans de tels cas d'abus, les salariés seront obligés d'aller en justice pour protéger et faire valoir leurs droits.

Par ailleurs, le législateur néglige complètement les risques psychosociaux liés au télétravail. De fait, il est possible d'exercer le télétravail sur une base permanente. Et celui-ci n'est limité par aucune condition. Le salarié est privé du droit d'influer sur le changement des règles déjà établies régissant son télétravail, et d'exiger un lieu de travail sur site. L'employeur n'a pas d'obligations supplémentaires de prévention des risques psychosociaux liés à l'isolement et de protection du télétravailleur en termes de soutien psychologique. Les contraintes de l'employeur dans ce domaine sont réduites au minimum.

II reste donc beaucoup de chemin à parcourir pour que tous ces risques soient pris en compte par l'employeur. Ceci devra très certainement passer par une réforme législative à terme. 